Editorial

\title{
Adolescent avoidance and risk behavior
}

Volume I Issue 4 - 2014

\section{Editorial}

It is well-known that adolescents evidence a wide array of behaviors that place them at risk physically, emotionally, and legally. Risk factors such as a family history of trauma, neglect, substance abuse, and mental illness are associated with adolescent behavior problem behaviors. Yet, relatively little is known about how these variables are mediated by personality and, especially, by traits like resilience or the ability to manage adversity. Examination of these variables is essential to moving our understanding of adolescent behavior in a more complex, multidimensional way. One important study in particular merits attention because it addresses the complex relationship among risk factors and personality in a more sophisticated way. ${ }^{1}$

A group of 2500 adolescents between the ages of 13 and 19 were followed over a five year period. Data about risk factors, coping style, and behavior were collected. With respect to risk factors and problem behaviors, these investigators focused on educational underachievement, delinquency, substance use, sexual behavior. With respect to coping style, two general strategies were identified: avoidance and impulsivity. The findings were startling: Coping style was the best predictor of overall adjustment when examined relative to risk factors, even when these factors were combined into a single factor and evaluated jointly. Specifically, an avoidant style accounted for over one third of variance in problem/high risk behavior, a finding that was consistent across age, gender, and racial subgroups.

Avoidance is a familiar coping strategy in adolescence, often used to forestall the emergence of inner tension as well as dysphoric mood and disorganizing emotions. At its extreme, it prevents one from accessing more adaptive strategies for handling environmental adversity, increasing the likelihood of problem behaviors thereby. Paradoxically, many teenagers engage in high risk behavior to avoid the discomfort associated with effective problem-solving, diminishing opportunities for new learning and perspective-taking.

Foremost among the implications of this study is the vital importance of early therapeutic interventions that address avoidance. Effectiveness depends on helping teenager's accurately identify maladaptive coping patterns and supporting their efforts to manage their anxiety, stress, and disappointment. Not only do they need to experiment safely with potential solutions, but must fashion strategies that balance their needs for social acceptance with a respect for their bodies with a realistic approach to the future. Encouraging about this research is its view that personal history, even when it includes trauma, is not destiny. Even for the older adolescent, dysfunctional patterns of behavior can be altered and risk behavior decreased when avoidant coping strategies are modified.

\author{
Ronald C Naso \\ American Board and Academy of Psychoanalysis, USA
}

Correspondence: Ronald C Naso,American Board and Academy of Psychoanalysis, 2777 Summer Street, Suite 504B, Stamford, CT 06905, USA, Tel 203-325-366I, Fax 203-325-0I45, Email rcnphd@gmail.com

Received: July 10, 2014 | Published: July II, 2014

Review scenarios with an eye focused upon social successes and areas for improvement

Parents can pick from a multitude of situations that contain rich examples for children to learn from. Overnight guests who act entitled and unappreciative, peers who initiate "cold calls" as a way of reaching out before a trip both will be taking, or dinner conversations that are not particularly interesting to the child, are all fodder for real life "social studies". Challenge your child to learn from the obvious errors of their peers, remind your child of their emotionally flat response to past interpersonal encounters, and push your child to make the phone call they have been avoiding due to the dread of discomfort. Greater social confidence comes from expanding their comfort zone.

\section{Acknowledgments}

None.

\section{Conflicts of interest}

Author declares there are no conflicts of interest.

\section{Funding}

None.

\section{References}

1. Cooper ML, Wood PK, Orcutt HK, et al. Personality and the predisposition to engage in risky or problem behaviors during adolescence. J Pers Soc Psychol. 2013;84(2):390-410. 